\title{
ORIGINAL
}

\section{EFECTIVIDAD COMPARATIVA DEL SEGUIMIENTO REMOTO A PERSONAS CON MARCAPASOS CARDÍACOS FRENTE AL CONVENCIONAL: CALIDAD DE VIDA A LOS 6 MESES (*)}

\author{
Antonio López-Villegas (1,2,3), Daniel Catalán-Matamoros (4), Emilio Robles-Musso (5) y Salvador \\ Peiró (6,7).
}

(1) Division of Medicine. Nordland Hospital, Bodø. Norway.

(2) Institute of Clinical Medicine. Faculty of Health Sciences. University of Tromsø. Tromsø. Norway.

(3) Área de Gestión. Hospital de Poniente, El Ejido-Almería, España.

(4) Unidad de Comunicación en Salud. Universidad Carlos III de Madrid, Madrid. España.

(5) Unidad de Cuidados Intensivos. Hospital de Poniente. El Ejido-Almería. España.

(6) Área de Investigación en Servicios de Salud. FISABIO. Valencia. España.

(7) Red de Investigación en Servicios de Salud en Enfermedades Crónicas (REDISSEC). Valencia. España.

(*) Este proyecto ha sido parcialmente financiado por el European Economic Area Grant 008/ ABELCM/2014A de la convocatoria de investigación NILS Science and Sustainability Program, 2014.

Los autores declaran no tener ningún tipo de conflicto de intereses en relación con este proyecto. Los equipos comerciales y empresas citadas en el manuscrito no han tenido ningún papel en la financiación del estudio, su diseño, análisis u otras decisiones relacionadas con el mismo y se referencian exclusivamente a efectos de identificar los diferentes dispositivos utilizados, sin que ello implique una recomendación positiva o negativa por parte de los autores.

\section{RESUMEN}

Fundamentos: El uso del seguimiento remoto (SR) de personas portadoras de marcapasos (MP) es limitado en comparación con la modalidad hospitalaria (SH), siendo escasa la evidencia científica que muestre su efectividad. El objetivo de este estudio fue comparar la calidad de vida en personas con diferentes modalidades de seguimiento.

Métodos: Ensayo clínico controlado, no aleatorizado ni enmascarado, con recogida de datos pre y post-implante del marcapasos durante los 6 meses de seguimiento. Se seleccionó a todos los pacientes mayores de 18 años a los que se les implantó un marcapasos en el periodo de estudio $(n=83)$, siendo asignados al grupo SR $(n=30)$ o al grupo $\mathrm{SH}(\mathrm{n}=53)$, en función de sus características personales y preferencias. Se analizaron las características basales y número de visitas al hospital, y se administró el cuestionario $E u$ roQol-5D (EQ-5D) para evaluar la calidad de vida relacionada con la salud y el Duke Activity Status Index (DASI) para valorar la capacidad funcional.

Resultados: No hubo diferencias significativas entre ambos grupos en relación al análisis basal, EQ-5D (SR:0,7299; SH:0,6769) y DASI (SR:21,41; SH:19,99) iniciales. A los 6 meses la calidad de vida mejoró en ambos grupos (SR:0,8613; SH:0,8175; $\mathrm{p}=0,439)$ aun sin diferencias estadísticamente significativas entre ellos. La puntuación final en el DASI fue similar a la basal $(20,51$ vs 21,80$)$. El grupo de SR realizó menos visitas/ transmisiones por paciente $(1,57)$ que el grupo hospitalario $(1,96)$, la reducción relativa fue del $31 \%(\mathrm{p}=0,015)$.

Conclusiones: El seguimiento remoto de personas portadoras de marcapasos se podría considerar como una opción equivalente al hospitalario en relación a la calidad de vida y reduce el número de visitas al hospital

Palabras clave: Calidad de vida. Investigación de efectividad comparativa. Marcapasos cardíaco artificial. Estudios de seguimiento. Consulta remota. Telemedicina.

Correspondencia

Antonio López Villegas

Nordland Hospital

Prinsens Gate 164

Postbox $1480\left(\mathrm{n}^{\circ} 38\right)$

8005 Bodø, Norway.

antoniolopezvillegas@andaluciajunta.es

DOI:

\section{ABSTRACT \\ Comparative Effectiveness of Remote Monitoring of People with Cardiac Pacemaker versus Conventional: Quality of Life at the 6 Months}

Background: The use of remote follow-up (RF) of people with pacemakers (PM) is limited in comparison to the hospital modality (HS), being still poor the scientific evidence that shows their comparative effectiveness. The aim of this study was to compare the quality of life in individuals with different modalities of follow-up.

Methods: Controlled, not randomized nor masked clinical trial, with data collection at pre and post-implantation of pacemakers during the 6 months follow-up. All patients over 18 years-old who were implanted a PM during the study period were selected $(n=83)$, and they were assigned to RF $(n=30)$ or HF $(n=53)$ groups according to their personal characteristics and patient's preferences. Baseline characteristics and number of visits to the hospital were analysed, the EuroQol-5D (EQ5D) questionnaire was administered to evaluate the health-related quality of life, and Duke Activity Status Index (DASI) to assess the functional capacity.

Results: There were no significant differences between both groups in relation to the baseline analysis, EQ5D (RF:0.7299; HF:0.6769) and DASI (RF:21.41; HF:19.99). At 6 months the quality of life was improved in both groups (EQ5D RF:0.8613; HF:0.8175; $\mathrm{p}=0,439$ ) still without significant differences between them. DASI score was similar to baseline (20.51 vs 21.80). RF group performed less transmissions/visits per patient (1.57) than hospital group (1.96; relative reduction $31 \%$; $\mathrm{p}=0.015$ ).

Conclusions: Remote follow-up of people with pacemakers might be considered as an equivalent option to the hospital follow-up in relation to the quality of life and it reduces the number of hospital visits.

Keywords: Quality of life. Comparative effectiveness research. Artificial pacemakers. Follow-up studies. Remote consultation. Telemedicine. 


\section{INTRODUCCIÓN}

Los marcapasos cardíacos (MP) han sido utilizados durante décadas para tratar las disfunciones del nodo sinusal y otras alteraciones del sistema de conducción cardíaco. Según el Registro Español de Marcapasos, durante 2013 se implantaron en España 36.042 mp, incluyendo recambios, $(755$ por millón de habitantes), más de la mitad a causa de bloqueos auriculo-ventriculares ${ }^{1}$. El seguimiento remoto (SR) de estos dispositivos y el control de los posibles problemas cardiacos es una parte integral de la atención de las personas con un implante ${ }^{2}$ y actualmente supone una carga de trabajo importante para los servicios de cardiología, así como de tiempo y económica para los pacientes que deben desplazarse a estas consultas para las sucesivas revisiones. El seguimiento remoto (SR) de estas personas -que incluye la transmisión de datos sobre el funcionamiento del dispositivo, episodios cardiovasculares, incluidos los eventos adversos ${ }^{3}$ (EA) y, en algunos casos, ciertos aspectos sobre el estado del paciente (como datos tempranos de descompensación cardíaca, impedancia transtorácica, peso, etcétera)- tiene ventajas y desventajas $^{3-6}$ pero podría suponer una ayuda importante para reducir las cargas tanto a los profesionales sanitarios como a los pacientes.

Con más de 300.000 unidades implantadas en todo el mundo desde que en 2001 se implantara la primera unidad en Europa, el SR de personas con MP es una tecnología consolidada. En el año 2013 se incluyó en programas de seguimiento o monitorización domiciliaria al $6 \%$ del total de personas con mp convencionales implantados en españa frente al $39 \%$ en el caso de personas con dispositivos de terapia de resincronización cardíaca (TRC) ${ }^{1}$. Esta discreta utilización puede estar relacionada con una menor evidencia científica sobre la efectividad, seguridad y coste-efectividad del SR en el caso de los MP, al menos por comparación con la trc, especialmente en lo referente a resultados reportados por los pacientes como los de calidad de vida relacionada con la salud (CVRS) y los de capacidad funcional.
El objetivo del estudio fue comparar la calidad de vida relacionada con la salud y la capacidad funcional en sujetos con marcapasos y seguimiento remoto respecto a los que tuvieron monitorización convencional en el hospital.

\section{MATERIAL Y MÉTODO}

Diseño. Ensayo clínico pragmático, controlado, no aleatorizado y no enmascarado, con asignación de los pacientes a SH o SR mediante transmisión electrónica de datos durante 6 meses de seguimiento desde la fecha del implante.

Entorno. El estudio se desarrolló en el Hospital de Poniente (El Ejido-Almería, España), un centro comarcal que atiende a una población de 256.000 habitantes y que realiza entre 80 y 90 implantes anuales.

Población. Todos los pacientes a los que se implantó un marcapasos entre el 01/10/2012 y el 30/11/2013, que cumplieran todos los criterios de inclusión y ninguno de los de exclusión. Criterios de inclusión: 1) ser mayor de 18 años, 2) haber recibido el implante de un marcapasos y 3) comprender y ser capaz de realizar adecuadamente la auto-monitorización domiciliaria o disponer de una persona cuidadora que pudiera hacerla. Criterios de exclusión: 1) estar participando en otro estudio, 2) tener implantado cualquier otro tipo de dispositivo cardíaco diferente al marcapasos, 3) no aceptar la participación en el estudio.

Dispositivos. Sistemas de estimulación con generador de marcapasos monocameral o bicameral (Medtronic) de los siguientes tipos: 1) Monocameral (vvi-vvir), formado por un generador y un único cable. 2) Bicameral de un solo cable (vdd). 3) Bicameral de dos cables (ddd). Todos los dispositivos incorporaban la tecnología para el SR (Monitor Carelink $($ ), pudiéndose activar o no en función del grupo de seguimiento al que fueran asignados los pacientes. 
Seguimiento inmediato post-implante y grupos de intervención y control. Tras el implante del marcapasos, todos los pacientes fueron hospitalizados durante 24-72 horas para observación y asistieron a una primera visita de seguimiento en el hospital a los 7-10 días tras el alta, durante la cual se revisó el correcto funcionamiento del dispositivo ajustándolo a las necesidades de cada paciente. Al mes del implante todos los pacientes realizaron una segunda visita al hospital en la que, tras revisar el correcto funcionamiento del dispositivo, el cardiólogo explicó a los pacientes las características, ventajas y desventajas de ambas modalidades de seguimiento y, siempre que cumplieran los criterios de inclusión, se les ofrecía la posibilidad de elegir entre ambas alternativas de seguimiento. El grupo de intervención quedó configurado por los pacientes que optaron por la SR, en cuyo caso el cardiólogo ajustó los correspondientes parámetros del marcapasos, instruyó al paciente en el uso de la tecnología y solicitó a la empresa proveedora del servicio el envío al domicilio del paciente del monitor correspondiente. El grupo control quedó configurado por los pacientes que optaron por el SH. Por lo tanto, la asignación a cada grupo no fue aleatoria sino voluntaria por parte de las personas participantes en la investigación.

Medida principal de resultados. la CVRS a los 6 meses de seguimiento medida a través de las utilidades de la versión española del euroqol-5d (EQ5D) ${ }^{7,8}$ (rango entre 0: muerte, y 1: mejor estado de salud posible, aunque se pueden tener puntuaciones negativas para estados "peores" que la muerte).

Medidas de resultado secundarias. La CVRS a los 6 meses de seguimiento medida mediante la escala visual analógica (EVA) del EQ5D valorada por el propio paciente (entre 0, peor estado de salud posible, y 100 , mejor estado de salud posible $)^{7,8}$. Capacidad funcional a los 6 meses de seguimiento valorada a través de la versión reducida del cuestionario Duke Activity Status Index (DASI) ${ }^{9,10}$ (rango entre 33: mejor estado funcional y 11,5 : peor estado funcional). También se registraron los valores en las diferentes dimensiones del EQ5D, los eventos cardiovasculares durante el seguimiento (definidos por la presencia de fibrilación auricular (FA)/taquiarritmia, la duración de los episodios dividida a su vez en las categorías de $>4$ horas y $<$ de 4 horas $\mathrm{y}$, finalmente episodios de FA alta o rápida $\mathrm{y}$ número de visitas o transmisiones realizadas durante el seguimiento.

Otras variables que se tuvieron en cuenta fueron la edad del paciente en el momento del ingreso para el implante (en algunos análisis se estratificó en personas hasta 79 años y personas de 80 y más años), el sexo, síntomas principales (síncope, mareo, disnea, angina), procedencia (urgencias, servicio de cardiología, otros servicios), indicación (enfermedad del seno, bloqueo auriculo-ventricular, otras indicaciones), tipo de estimulación, CVRS basal y al mes de seguimiento y capacidad funcional basal y al mes de seguimiento medida mediante el EQ5D y capacidad funcional basal y al mes de seguimiento medida a través del DASI.

El tamaño muestral del estudio, condicionado por la capacidad de seleción de pacientes del centro, se estimó en 90 pacientes (45 por grupo) para detectar una diferencia entre grupos en la variable principal del estudio (utilidades del EQ5D) de 0,12 puntos (desde 0,68 a 0,80 asumiendo una desviación estándar de 0,20 ) con un error alfa de 0,05 y un poder del 0,80 .

Seguimiento. A todos los participantes se les realizaron 3 entrevistas (basal, al mes -antes de su elección por una u otra modalidad de monitorización-y a los 6 meses del implante) durante las cuales se cumplimentaron los cuestionarios. Estas entrevistas se realizaron de forma personalizada durante la hospitalización y las visitas programadas, y mediante entrevista telefónica a los 6 meses.

Aspectos éticos. El estudio fue aprobado por el Comité de Ética de Investigación de Centro-Almería y se aplicó desarrollando los 
preceptos de ética e investigación marcados por la Declaración de Helsinki. Todos los pacientes firmaron el correspondiente consentimiento informado previamente a su inclusión en el estudio y se adoptaron las medidas oportunas para garantizar la privacidad de los datos identificativos de los pacientes. El ensayo clínico quedó registrado como ClinicalTrials. gov Identifier. NCT02234245.

Análisis estadístico. En primer lugar se analizaron las características basales de los pacientes y las posibles diferencias entre grupos utilizando la prueba de $\chi^{2}$ (sustituida por la prueba exacta de Fisher cuando el número de casos era inferior a 5) para las variables cualitativas y la prueba de diferencia de medias para las cuantitativas. También se analizaron las diferencias intragrupo entre las valoraciones basal, al mes y a los 6 meses, utilizando la prueba de diferencia de medias para datos apareados. Seguidamente se analizaron las diferencias crudas entre grupos en la variable principal del estudio (puntuación de utilidad en el EQ5D) y las variables secundarias (puntuación de calidad en la eva y puntuación de capacidad funcional en el dasi utilizando en todos los casos la prueba de diferencia de medias. los resultados se presentan incluyendo los respectivos intervalos de confianza al 95\% (IC95). Finalmente, se analizó la asociación entre el tipo de seguimiento y los resultados en la variable principal del estudio. Para ello, dado que la asignación de los participantes a las diferentes alternativas de seguimiento no fue aleatoria conllevando la posibilidad de sesgo de indicación (los pacientes podrían haber escogido a una $u$ otra alternativa en función de características que, a su vez, se asociaran a la calidad de vida a los 6 meses) se realizó una regresión logística para identificar los factores asociados a la indicación del tipo de seguimiento con el fin de utilizarlos como propensity score matching para ajustar el resultado de la variable principal. Finalmente, se realizó una regresión lineal múltiple para identificar los factores asociados independientemente a las utilidades del EQ5D a los 6 meses y, específicamente, si el tipo de segui- miento era uno de estos factores. Todos los análisis se realizaron utilizando el programa STATA (College Station, Texas) v13.

\section{RESULTADOS}

De los 123 participantes que recibieron el implante de un marcapasos en el periodo de estudio, 29 fueron excluidos en base a los criterios de inclusión/exclusión, y 5 no dieron su consentimiento para ser incluidos en el estudio (figura 1). Optaron por el seguimiento remoto 33 sujetos mientras que 56 prefirieron el seguimiento en el hospital. Durante la investigación, 5 pacientes abandonaron voluntariamente y 1 falleció antes de la entrevista programada a los 6 meses, quedando un total de 83 pacientes para el análisis (30 en el grupo SR y 53 en el grupo SH).

La edad media de los pacientes fue de 77,5 años (IC95\%: 76,0-79,1), el 78,3\% eran hombres. La sintomatología más frecuente fue el síncope $(56,6 \%)$ seguida de los mareos. El $60 \%$ de los pacientes procedían del servicio de cardiología y la indicación más frecuente fue el bloqueo auriculo-ventricular $(65,0 \%)$ seguida de la enfermedad del seno $(18,1 \%)$. En el 75,9\% de los casos se emplearon marcapasos bicamerales. No se hallaron diferencias estadísticamente significativas entre los grupos de SR y SH en ninguna de las variables evaluadas (tabla 1).

En el momento del ingreso hospitalario los pacientes tuvieron una puntuación media de 0,6961 (IC95\%: 0,6135-0,7787) en el EQ5D y de 58,31 $(53,23-63,39)$ en la EVA, que ascendieron a $0,7216(0,6508-0,7923)$ y $63,61(60,29-66,93)$ respectivamente al mes del implante. Los cambios en los resultados del EQ5D basal y al mes no fueron estadísticamente significativos $(p=0,594)$, pero sí en el caso de la EVA $(p=0,042)$. Los grupos SR y SH no mostraron diferencias estadísticamente significativas en las puntuaciones del EQ5D y la EVA al ingreso ni al mes (tabla 2), ni en ninguna de las dimensiones del EQ5D al ingreso (tabla 3). Respecto al DASI, los pacientes tuvieron una puntuación media 
Figura 1

Diagrama de seguimiento de las personas portadoras de marcapasos cardíaco

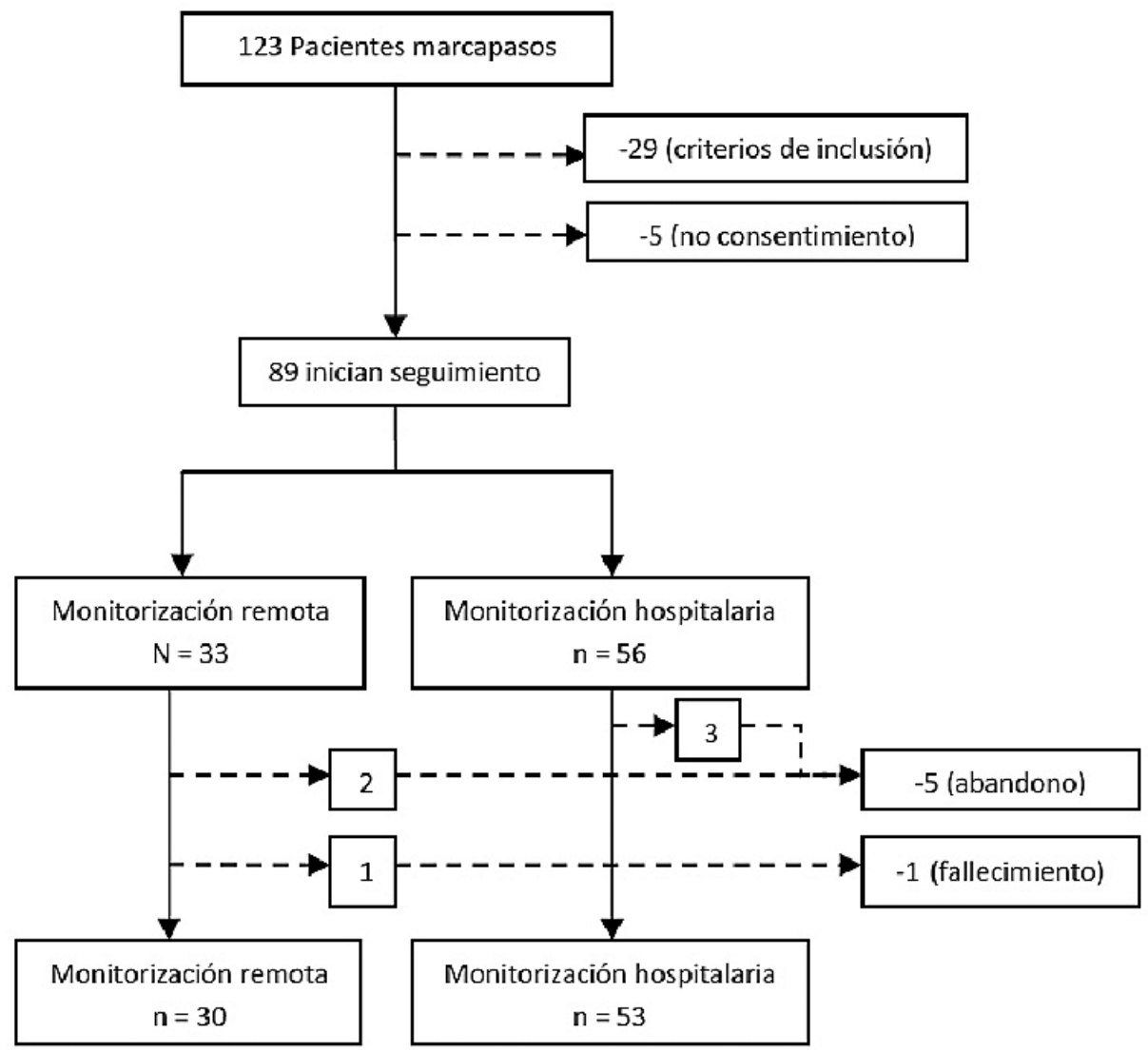

al ingreso de 20,51 (IC95\%: 19,25-21,76) y 19,24 (IC95\%: 18,01-20,47) en la valoración al mes $(\mathrm{p}=0,052)$. No se hallaron diferencias estadísticamente significativas en las puntuaciones del DASI entre los grupos SR y SH $(21,42$ vs 19,99; $\mathrm{p}=0,279)$ al ingreso o al mes de seguimiento $(20,60$ vs 18,$47 ; \mathrm{p}=0,099)$ (tabla 3).

En promedio se detectaron 0,64 (IC95\%: 0,46-0,81) eventos cardiovasculares por paciente en los 6 meses de seguimiento, sin diferencias entre el grupo SR $(0,53$; IC $95 \%$ : $0,24-0,82)$ y el grupo SH $(0,70$; IC $95 \%$ : 0,47 $0,92)$. No se pudo obtener información sobre la causa de muerte del paciente fallecido, aunque no consta que solicitara asistencia por problemas cardiológicos. En cuanto a cargas de trabajo - se contabilizaron todas las visitas/transmisiones que realizaron los pacientes desde la fecha del implante (7-10 días, meses 1 y 6 ( i correspondía) y, aquellas otras realizadas de manera no programada a la consulta de cardiología) - produciéndose de media 1,81 visitas/transmisiones por paciente, 1,57 (IC95\%: 1,31-1,82) en el grupo remoto frente a 1,96 (IC95\%:1,77-2,16) en el grupo hospitalario (diferencia: -0,39 visitas/ paciente; $p=0,015$ ). 


\begin{tabular}{|c|c|c|c|c|c|c|}
\hline \multicolumn{7}{|c|}{$\begin{array}{c}\text { Tabla } 1 \\
\text { Características basales de los pacientes } \\
\text { en los grupos con seguimiento remoto } \\
\text { y hospitalario }\end{array}$} \\
\hline & & \multicolumn{2}{|c|}{$\begin{array}{l}\text { Seguimiento } \\
\text { remoto }\end{array}$} & \multicolumn{2}{|c|}{$\begin{array}{l}\text { Seguimiento } \\
\text { hospitalario }\end{array}$} & \multirow[t]{2}{*}{$\mathrm{p}$} \\
\hline & & $\mathrm{n}$ & $\%$ & $\mathrm{n}$ & $\%$ & \\
\hline \multirow{2}{*}{ Edad } & $<=79$ & 16 & 53,33 & 31 & 58,49 & \multirow{2}{*}{0,65} \\
\hline & $80+$ & 14 & 46,67 & 22 & 41,51 & \\
\hline \multirow{2}{*}{ Sexo } & Hombre & 21 & 70,00 & 44 & 83,02 & \multirow{2}{*}{0,17} \\
\hline & Mujer & 9 & 30,00 & 9 & 16,98 & \\
\hline \multirow{4}{*}{ Síntomas } & Síncope & 19 & 63,33 & 27 & 50,94 & \multirow{4}{*}{0,78} \\
\hline & \begin{tabular}{|l|} 
Mareo \\
\end{tabular} & 7 & 23,33 & 18 & 33,96 & \\
\hline & Disnea & 2 & 6,67 & 5 & 9,43 & \\
\hline & \begin{tabular}{|l} 
Angina \\
\end{tabular} & 2 & 6,67 & 3 & 5,67 & \\
\hline \multirow{3}{*}{ Procedencia } & Urgencias & 6 & 20,00 & 9 & 16,98 & \multirow{3}{*}{0,92} \\
\hline & Cardiología & 18 & 60,00 & 32 & 60,38 & \\
\hline & Otros servicios & 6 & 20,00 & 12 & 22,64 & \\
\hline \multirow{3}{*}{ Indicación } & Enf. Seno & 4 & 13,33 & 11 & 20,75 & \multirow{3}{*}{0,68} \\
\hline & Bloqueo AV & 21 & 70,00 & 33 & 62,27 & \\
\hline & Otras & 5 & 16,67 & 9 & 16,98 & \\
\hline \multirow{4}{*}{ Estimulación } & VVI & 3 & 10,00 & 5 & 9,43 & \multirow{4}{*}{0,36} \\
\hline & VVIR & 2 & 6,67 & 10 & 18,87 & \\
\hline & VDD & 4 & 13,33 & 6 & 11,32 & \\
\hline & \begin{tabular}{|l} 
DDR \\
\end{tabular} & 21 & 70,00 & 32 & 60,38 & \\
\hline Total & & 30 & 100 & 53 & 100 & \\
\hline
\end{tabular}

La $\mathrm{p}$ corresponde a la prueba de $\chi^{2}$ (sustituida por la prueba exacta de Fisher si alguna celdilla contenía $<5$ casos). AV: Auriculo-ventricular; DDD-DDR: Bicameral de dos cables; VDD: Bicameral de un solo cable; VVI-VVIR: Monocameral de un solo cable.

\section{Tabla 2}

\section{Calidad de vida basal y al mes de los pacientes} en seguimiento remoto y hospitalario

\begin{tabular}{|c|c|c|c|c|c|c|}
\hline \multirow{2}{*}{\multicolumn{2}{|c|}{ Dimensiones EQ5D }} & \multicolumn{2}{|c|}{$\begin{array}{l}\text { Seguimiento } \\
\text { remoto }\end{array}$} & \multicolumn{2}{|c|}{$\begin{array}{l}\text { Seguimiento } \\
\text { hospitalario }\end{array}$} & \multirow[t]{2}{*}{$\mathrm{p}$} \\
\hline & & $\mathrm{n}$ & $\%$ & $\mathrm{n}$ & $\%$ & \\
\hline \multirow{3}{*}{ Movilidad } & Sin problemas & 19 & 63,33 & 30 & 56,60 & \multirow{3}{*}{0,48} \\
\hline & \begin{tabular}{|l|} 
Alguno \\
\end{tabular} & 8 & 26,67 & 20 & 37,74 & \\
\hline & Encamado & 3 & 10,00 & 3 & 5,66 & \\
\hline \multirow{3}{*}{$\begin{array}{l}\text { Cuidado } \\
\text { personal }\end{array}$} & Sin problemas & 23 & 76,67 & 36 & 67,93 & \multirow{3}{*}{0,38} \\
\hline & \begin{tabular}{|l|} 
Alguno \\
\end{tabular} & 6 & 20,00 & 10 & 18,80 & \\
\hline & Incapaz & 1 & 3,33 & 7 & 13,21 & \\
\hline \multirow{3}{*}{$\begin{array}{l}\text { Actividad } \\
\text { cotidiana }\end{array}$} & Sin problemas & 23 & 76,67 & 31 & 58,49 & \multirow{3}{*}{0,31} \\
\hline & \begin{tabular}{|l} 
Alguno \\
\end{tabular} & 5 & 16,66 & 14 & 26,42 & \\
\hline & Incapaz & 2 & 6,67 & 8 & 15,09 & \\
\hline \multirow{3}{*}{$\begin{array}{l}\text { Dolor/ } \\
\text { malestar }\end{array}$} & No & 18 & 60,00 & 31 & 58,49 & \multirow{3}{*}{1,00} \\
\hline & \begin{tabular}{|l|} 
Moderado \\
\end{tabular} & 11 & 36,67 & 20 & 37,74 & \\
\hline & Mucho & 1 & 3,33 & 2 & 3,77 & \\
\hline \multirow{3}{*}{$\begin{array}{l}\text { Ansiedad/ } \\
\text { depresión }\end{array}$} & No & 21 & 70,00 & 33 & 62,26 & \multirow{3}{*}{0,27} \\
\hline & \begin{tabular}{|l|} 
Moderado \\
\end{tabular} & 8 & 26,67 & 20 & 37,74 & \\
\hline & \begin{tabular}{|l|} 
Mucho \\
\end{tabular} & 1 & 3,33 & 0 & 0,00 & \\
\hline \begin{tabular}{|l|} 
Total \\
\end{tabular} & & 30 & 100 & 53 & 100 & \\
\hline \begin{tabular}{|l|}
$\begin{array}{l}\text { EQ5D total } \\
\text { (ingreso) }\end{array}$ \\
\end{tabular} & (media) & 30 & 0,73 & 53 & 0,68 & 0,54 \\
\hline \begin{tabular}{|l} 
EQ5D EVA \\
(ingreso)
\end{tabular} & (media) & 30 & 59,00 & 53 & 57,92 & 0,84 \\
\hline EQ5D total mes & (media) & 30 & 0,76 & 53 & 0,70 & 0,42 \\
\hline EQ5D EVA mes & (media) & 30 & 65,66 & 53 & 62,45 & 0,36 \\
\hline
\end{tabular}

La $\mathrm{p}$ corresponde a la prueba de $\chi^{2}$ (sustituida por la prueba exacta de Fisher si alguna celdilla contenía $<5$ casos), excepto para los EuroQol-5D total y EVA que corresponde a la prueba de diferencia de medias. EQ5D: EuroQol-5D. EVA: Escala Visual Analógica.
A los 6 meses de seguimiento, la puntuación de utilidad en el EQ5D para el conjunto de participantes ascendió hasta 0,8334 (IC95\%: 0,7796-0,8871), 0,1373 puntos más que la basal (incremento relativo del $19,7 \% ; \mathrm{p}=0,003)$ y 0,1118 puntos más que la puntuación al mes del implante (incremento relativo del $15,5 \%$; $<<0,0001)$. Respecto a la EVA, la puntuación a los 6 meses alcanzó los 68,73 puntos (IC95\%: 66,00$71,47) 10,42$ puntos más que al ingreso (incremento relativo del $17,9 \% ; \mathrm{p}=0,0003$ ) y 5,12 puntos más que al mes del implante (incremento relativo del $8,1 \% ; \mathrm{p}<0,0001$ ). La puntuación en el DASI a los 6 meses fue de 21,80 (IC95\%: 20,60-23,00), sin diferencias estadísticamente significativas respecto al ingreso, y de 2,6 puntos menor que al mes del implante (incremento del 13,3\%; $\mathrm{p}<0,0001)$. Ningún grupo de seguimiento presentó diferencias estadísticamente significativas en ninguno de los análisis realizados (tabla 4).

\begin{tabular}{|c|c|c|c|c|c|c|}
\hline \multicolumn{7}{|c|}{$\begin{array}{c}\text { Tabla } 3 \\
\text { Capacidad funcional DASI basal } \\
\text { y al mes de los pacientes en seguimiento } \\
\text { remoto y hospitalario }\end{array}$} \\
\hline & & \multicolumn{2}{|c|}{$\begin{array}{l}\text { Seguimiento } \\
\text { remoto }\end{array}$} & \multicolumn{2}{|c|}{$\begin{array}{l}\text { Seguimiento } \\
\text { hospitalario }\end{array}$} & \multirow[t]{2}{*}{$\mathrm{p}$} \\
\hline & & $\mathrm{n}$ & $\%$ & $\mathrm{n}$ & $\%$ & \\
\hline \multirow{3}{*}{$\begin{array}{l}\text { Caminar } \\
\text { en casa }\end{array}$} & No & 1 & 3,33 & $\frac{11}{2}$ & 3,77 & \multirow{3}{*}{0,80} \\
\hline & Con dificultad & 4 & 13,33 & 211 & $\begin{array}{r}5,71 \\
20,75\end{array}$ & \\
\hline & Sin dificultad & 25 & 83,34 & 40 & 75,48 & \\
\hline \multirow{3}{*}{$\begin{array}{l}\text { Caminar } \\
1-2 \\
\text { manzanas }\end{array}$} & No & 2 & 6,67 & 9 & 17,98 & \multirow{3}{*}{0,23} \\
\hline & Con dificultad & 9 & 30,00 & 9 & 17,98 & \\
\hline & Sin dificultad & 19 & 63,33 & 35 & 66,04 & \\
\hline \multirow{3}{*}{$\begin{array}{l}\text { Sube/baja } \\
\text { escalon }\end{array}$} & No & 4 & 13,33 & 15 & 28,30 & \multirow{3}{*}{0,22} \\
\hline & Con dificultad & 15 & 50,00 & 18 & 34,96 & \\
\hline & Sin dificultad & 11 & 36,67 & 20 & 37,74 & \\
\hline \multirow{3}{*}{\begin{tabular}{|l|} 
Correr \\
distancias \\
cortas
\end{tabular}} & No & 26 & 86,87 & 49 & 92,45 & \multirow{3}{*}{0,28} \\
\hline & Con dificultad & 1 & 3,33 & 3 & 5,66 & \\
\hline & Sin dificultad & 3 & 10,00 & 1 & 1,89 & \\
\hline \multirow{3}{*}{$\begin{array}{l}\text { Trabajo } \\
\text { casa ligero }\end{array}$} & No & 9 & 30,00 & 23 & 43,40 & \multirow{3}{*}{0,48} \\
\hline & Con dificultad & 4 & 13,33 & 6 & 11,32 & \\
\hline & Sin dificultad & 17 & 56,67 & 24 & 45,28 & \\
\hline \multirow{3}{*}{\begin{tabular}{|l|} 
Trabajo \\
casa \\
moderado \\
\end{tabular}} & No & 14 & 46,67 & 28 & 52,83 & \multirow{3}{*}{0,73} \\
\hline & Con dificultad & 5 & 16,67 & 6 & 11,32 & \\
\hline & Sin dificultad & 11 & 36,67 & 19 & 35,85 & \\
\hline \multirow{3}{*}{$\begin{array}{l}\text { Trabajo } \\
\text { casa } \\
\text { pesado }\end{array}$} & No & 16 & 53,33 & 36 & 67,93 & \multirow{3}{*}{0,36} \\
\hline & Con dificultad & 8 & 26,67 & 8 & 15,09 & \\
\hline & Sin dificultad & 6 & 20,00 & 9 & 17,98 & \\
\hline \multirow{3}{*}{$\begin{array}{l}\text { Ejercicio } \\
\text { intenso }\end{array}$} & No & 21 & 70,00 & 40 & 75,48 & \multirow{3}{*}{0,92} \\
\hline & Con dificultad & 2 & 6,67 & 3 & 5,65 & \\
\hline & Sin dificultad & 7 & 23,33 & 10 & 18,87 & \\
\hline Total (ingreso) & (media) & 30 & 21,42 & 53 & 19,99 & 0,280 \\
\hline Total (mes) & (media) & 30 & 20,60 & 53 & 18,47 & 0,10 \\
\hline
\end{tabular}

La p corresponde a la prueba de $\chi 2$ (sustituida la prueba exacta de Fisher si alguna celdilla contenía $<5$ casos) excepto para el Indice de Actividad de Duke total que corresponde a la diferencia de medias. dasi: Indice de Actividad de Duke. 


\begin{tabular}{|c|c|c|c|c|c|}
\hline \multicolumn{6}{|c|}{$\begin{array}{c}\text { Tabla } 4 \\
\text { Resultados de calidad vida y capacidad } \\
\text { funcional a los } 6 \text { meses }\end{array}$} \\
\hline & \multicolumn{2}{|c|}{$\begin{array}{l}\text { Seguimiento } \\
\text { remoto }\end{array}$} & \multicolumn{2}{|c|}{$\begin{array}{l}\text { Seguimiento } \\
\text { hospitalario }\end{array}$} & \multirow{2}{*}{$\mathrm{p}$} \\
\hline & Media & $\mathrm{IC}_{95}$ & Media & $\mathrm{IC}_{95}$ & \\
\hline DASI & 86,13 & $0,77-0,95$ & 0,82 & $0,75-0,89$ & 0,44 \\
\hline EQ5D (EVA) & \begin{tabular}{|l|}
69,50 \\
\end{tabular} & $64,89-74,10$ & 68,30 & $64,78-71,81$ & 0,68 \\
\hline DASI & 23,10 & $21,20-25,10$ & 21,07 & $19,50-22,60$ & 0,10 \\
\hline
\end{tabular}

La $\mathrm{p}$ corresponde a la prueba de diferencia de medias. EQ5D EuroQoL-5D; EVA: Escala Visual Análoga; DASI: Índice de Actividad de Duke.

En el modelo de regresión logística construido para identificar factores asociados a la decisión de los pacientes de seguir uno u otro tipo de seguimiento (tabla 5), ninguna de las características de los pacientes disponibles ni su puntuación de calidad de vida en el momento de la elección se asoció a la decisión de optar por una u otra alternativa con los niveles de significación habituales $(\mathrm{p}<0,05)$. El modelo retuvo como variables más explicativas la puntuación DASI al mes (la probabilidad de optar por la SR creció un $0,07 \%$ por cada punto adicional-empeoramiento- en el DASI) y el sexo (los hombres tuvieron una probabilidad mayor en algo más del doble que las mujeres de recibir SR) (ambas con $\mathrm{p}>0,10$ ). La capacidad explicativa del modelo fue muy discreta [Area Under Curve $(\mathrm{AUC})=0,63)]$ aunque la calibración fue correcta $\left[\mathrm{p}\left(\chi^{2}\right.\right.$ Hosmer-Lemeshow $)$ : $0,339]$. Siguiendo el plan de análisis, las probabilidades predichas por este modelo se retuvie-

\begin{tabular}{|c|c|c|c|}
\hline \multicolumn{4}{|c|}{$\begin{array}{c}\text { Tabla } 6 \\
\text { Factores asociados a la puntuación } \\
\text { en el EQ5D a los } 6 \text { meses } \\
\text { (regresión lineal múltiple) }\end{array}$} \\
\hline & Coef B & IC95 Coef B & $\mathrm{p}$ \\
\hline Sexo mujer $v s$ hombre & $-0,0736$ & $-0,1500 ; 0,0027$ & $<0,001$ \\
\hline Procedencia "Otros Servicios" vs urgencias & $-0,0779$ & $-0,1555 ;-0,0002$ & 0,049 \\
\hline Síntomas: Angina $v s$ síncope & $-0,1321$ & $-0,2682 ; 0,0039$ & 0,057 \\
\hline Indicación: BAV vs enfermedad seno & 0,1790 & 0,$0905 ; 0,2675$ & $<0,001$ \\
\hline Indicación: Otras $v s$ enfermedad s & 0,1954 & 0,$0910 ; 0,2998$ & $<0,001$ \\
\hline EQ5D al mes por punto & 0,4674 & 0,$3595 ; 0,5753$ & $<0,001$ \\
\hline Constante & 0,3875 & 0,$2893 ; 0,4856$ & $<0,001$ \\
\hline
\end{tabular}

$\mathrm{n}=83 ; \mathrm{r}^{2}: 0,686 ; \mathrm{p}<0,001$; Variables no incorporadas al modelo por falta de significación estadística $[\mathrm{p}($ enter $)>0,05$ o $\mathrm{p}($ remove $)>0,10]$ : propensity score respecto al tipo de seguimiento, modelo de marcapasos, puntuación en la escala DASI, grupo de edad, procedencia del servicio de cardiología ( vs urgencias), síntomas de mareos o disnea (vs síncope), tipo de seguimiento.

BAV: Bloqueo auriculo-ventricular. EQ5D: EuroQol-5D. DASI: Indice de Actividad de Duke

\begin{tabular}{|c|c|c|c|}
\hline \multicolumn{4}{|c|}{$\begin{array}{c}\text { Tabla } 5 \\
\text { Factores asociados a recibir seguimiento } \\
\text { remoto (regresión logística) }\end{array}$} \\
\hline & OR & $\mathrm{IC}_{95} \mathrm{OR}$ & $\mathrm{p}$ \\
\hline DASI $1^{\text {er }}$ mes por punto & 1,07 & $0,99-1,16$ & 0,10 \\
\hline Sexo hombre $v$. mujer & 2,14 & $0,72-6,35$ & 0,17 \\
\hline \begin{tabular}{|l} 
Constante \\
\end{tabular} & 0,12 & $0,02-0,69$ & 0,02 \\
\hline
\end{tabular}

La p corresponde a la prueba de diferencia de medias. EQ5D: EuroQoL-5D; EVA: Escala Visual Análoga; DASI: Índice de Actividad de Duke.

ron para ajustar la regresión múltiple respecto a la puntuación final en el EQ5D (propensity score matching).

En el análisis multivariable (tabla 6), ser mujer (frente a hombre), la procedencia de otros servicios ( $v s$ urgencias) y los síntomas de angina (frente al síncope) empeoraron discretamente la puntuación en el EQ5D a los 6 meses, mientras que la indicación en bloqueo auriculo-ventricular y otras indicaciones ( $v S$ enfermedad del seno) y, sobre todo, la mejor puntuación al mes de la implantación, se asociaron a mejores puntuaciones en el EQ5D a los 6 meses. No se hallaron diferencias en el EQ5D a los 6 meses en función de si el seguimiento fue remoto u hospitalario.

\section{DISCUSIÓN}

Los resultados de este estudio muestran, en primer lugar, la ausencia de diferencias en la calidad de vida a los 6 meses de seguimiento entre los participantes que tuvieron visitas programadas en el hospital y los que utilizaron el seguimiento remoto con dispositivos de transmisión electrónica. Los análisis secundarios apoyan este resultado al no encontrar diferencias entre ambos grupos en la CVRS valorada por el propio paciente (EVA) o en la capacidad funcional valorada por el DASI. Estos resultados son compatibles con los del estudio COMPAS ${ }^{11}$, un ensayo clínico con selección aleatoria de 538 sujetos con implantes a SR o SH que utilizó el cuestionario SF-36, no encontrando diferencias entre ambos grupos en las puntuaciones de las dimensiones física (65 vs 67), psíquica (68 vs 71) y salud global (68 vs71). En conjunto, estos resultados refuerzan la ausencia de diferencias en efectividad y se- 
guridad del SR respecto al SH. Mientras que el estudio COMPAS no encontró una mejoría en la CVRS de los participantes -tanto del grupo intervención como del grupo control- entre el momento del reclutamiento (un mes antes del implante) y el final del seguimiento (18 meses en promedio $)^{11}$, en nuestro estudio los pacientes experimentaron una importante y significativa mejoría en las utilidades del EQ5D entre el momento del ingreso, al mes (tras el implante) y al final del seguimiento. La EVA del EQ5D se comportó de forma similar, con mejoras significativas entre las 3 mediciones, pero no así el DASI, que mostró puntuaciones muy cercanas en las 3 valoraciones realizadas. Las posibles explicaciones de estas diferencias entre estudios y entre los resultados ofrecidos por cada instrumento son muy especulativas e incluyen las diferencias en el entorno, en la sensibilidad de los cuestionarios y en el tiempo de seguimiento.

Los estudios que han evaluado el SR de dispositivos electrónicos implantables frente al SH, tanto marcapasos como desfibriladores automáticos implantables (DAI), le atribuyen una reducción de visitas médicas ${ }^{12-16}$, mejoras en seguridad ${ }^{11,17-24}$, mayor satisfacción de los pacientes $^{25-27} \mathrm{y}$, aunque apenas existen evaluaciones económicas, cierto potencial para producir ahorros ${ }^{28-30}$. Con todo, la mayor parte de estudios se refieren a DAI y prácticamente ninguno utilizó medidas de CVRS para valorar los resultados en pacientes con MP, a excepción del COMPAS. Pese al poco poder estadístico para su análisis, algunas de las variables secundarias de nuestro estudio sugieren cierta reducción del número de visitas y una capacidad de detección de eventos cardiovasculares al menos similar a la del grupo con sh.

La conclusión de no existir diferencias entre las dos alternativas de seguimiento viene matizada por algunas limitaciones. En primer lugar, se trata de un estudio no aleatorizado en el que los participantes decidían el tipo de seguimiento junto al cardiólogo. Aunque no se encuentran diferencias entre grupos en las variables basales valoradas, es posible que las haya en otras variables no observadas (nivel cultural, ruralidad/distancia al hospital, comorbilidad, fragilidad, etc.) que, a su vez, tuvieran influencia en el resultado final de CVRS (sesgo de indicación). Este aspecto se intentó abordar utilizando un ajuste multivariable por las características que determinaban la elección de uno u otro tipo de seguimiento (propensity score matching) pero el número de variables disponibles era limitado y es posible que no capturaran el efecto contextual de otras variables determinantes del resultado. En todo caso, la asignación consensuada con el paciente utilizada en nuestro estudio se aproxima a la práctica diaria $\mathrm{y}$, previsiblemente, los resultados obtenidos serán más cercanos a los esperables en el mundo real que los obtenidos con aleatorización (mayor validez externa a expensas de menor validez interna). En segundo lugar, y en este mismo sentido, se trata de un ensayo clínico abierto en el que tanto pacientes como profesionales conocían en qué alternativa estaba incluido cada sujeto, un aspecto que potencialmente podría influir en sus comportamientos y en su respuesta a las mediciones. En tercer lugar, el número de pacientes seleccionados para participar en el estudio -derivado de los condicionantes de estudio unicéntrico y con un limitado número de implantes anuales- permite un escaso poder para detectar diferencias entre grupos. De hecho, el cálculo inicial sólo hubiera permitido detectar como significativas diferencias de 0,12 puntos en el resultado principal (3 veces mayores que la diferencia mínima clínicamente relevante en el EuroQol-5D). El menor tamaño muestral final y las diferencias de número de sujetos en cada grupo habrán reducido algo más el poder estadístico real. Esto implica que pudieran existir diferencias relevantes entre ambas alternativas que no han podido ser detectadas en el actual estudio, aunque debe señalarse que la cercanía entre los valores de los resultados de ambos grupos en los diferentes análisis realizados, no sugiere la existencia de dichas diferencias.

En cuarto lugar, respecto a la generalización de los resultados a otros entornos, las 
características de la población atendida fueron muy similares (en edad, sexo, síntomas e indicaciones) a las reportadas en el Registro Español de Marcapasos ${ }^{8}$, aspecto que apoya la posibilidad de generalización. Quinto, en cuanto al seguimiento, el estudio actual tuvo una duración relativamente corta (6 meses), por lo que los resultados podrían cambiar con un seguimiento a más largo plazo (actualmente continúa). Finalmente, no se analizaron los eventos infrecuentes (muertes, hospitalizaciones, abandonos por efectos adversos, etc.) al no disponer de poder estadístico para su análisis. No obstante, la mayor parte fueron analizados en el COMPAS ${ }^{11}$, mostrando la equivalencia entre alternativas, incluso sugeriendo ciertas mejoras en los resultados clínicos de los sujetos con seguimiento remoto.

Teniendo en cuenta la incertidumbre derivada de las limitaciones reseñadas en el apartado anterior, el seguimiento remoto se configura como una opción equivalente al sh tradicional para mejorar la calidad de vida de los pacientes que reciben un marcapasos $\mathrm{y}$, adicionalmente, reduce significativamente el número de visitas al hospital. En este contexto de equivalencia, la generalización de esta tecnología en nuestro entorno (recordemos que en 2013 supuso el 6\% del total de implantes de $m p^{1}$ ) dependería sobre todo de su capacidad para reducir costes o cargas de trabajo para el Sistema Nacional de Salud y/o para los pacientes, factores que dependen no sólo de la tecnología empleada sino también de la organización del seguimiento adoptada por cada hospital, un aspecto -el organizativo- al que se le ha prestado muy escasa atención hasta la fecha.

\section{AGRADECIMIENTOS}

Los autores expresan su agradecimiento a María Antonia Contreras, Antonia Soria, Belén Campos, Jesús Ramos y Sonia García de la Unidad de Cuidados Intensivos del Hospital de Poniente, por su colaboración y apoyo en el desarrollo del proyecto.

\section{BIBLIOGRAFÍA}

1. Coma-Samartín R, Cano Perez O, Pombo Jiménez M. Registro Español de Marcapasos. XI Informe Oficial de la Sección de Estimulación Cardiaca de la Sociedad Española de Cardiología (2013). Rev Esp Cardiol. 2014;67(12):1024-38.

2. Maisel WH, Hauser RG, Hammill SC, Hauser RG, Ellenbogen KA, Epstein AE, et al. Recommendations from the Heart Rhythm Society Task Force on Lead Performance Policies and Guidelines: developed in collaboration with the American College of Cardiology (ACC) and the American Heart Association (AHA). Heart Rhythm. 2009;6(6):869-85.

3. Perl S, Stiegler P, Rotman B, Prenner G, Lercher P, Anelli-Monti M, et al. Socio-economic effects and cost saving potential of remote patient monitoring (SAVEHM trial). Int J Cardiol. 2013;169:402-407.

4. Varma N, Ricci RP. Telemedicine and cardiac implants: what is the benefit? Eur Heart J. 2013;34(25):1885-95.

5. Osca J, Sancho M, Navarro J, Cano O, Raso R, Castro $\mathrm{J}$, et al. Fiabilidad técnica y seguridad clínica de un sistema de monitorización remota de dispositivos cardiacos antiarrítmicos. Rev Esp Cardiol. 2009;62(08):886-95.

6. De Cock C, Elders J, van Hemel M, van den Broek K, van Erven L, de Mol B, et al. Remote monitoring and follow-up of cardiovascular implantable electronic devices in the Netherlands. An expert consensus report of the Netherlands Society of Cardiology. Neth Heart J. 2012;20(2):53-65.

7. EuroQol Group. EuroQol . A new facility for the measurement of health-related quality of life. Health Policy. 1990;16:199-208.

8. Badia X, Roset M, Montserrat S, Herdman M, Segura A. La versión española del EuroQol: descripción y aplicaciones. Med Clin (Barc). 1999;112(Supl 1):S79-86

9. Hlatky MA, Boineau RE, Higginbotham MB, Lee KL, Mark DB, Califf RM, et al. A brief self-administered questionnaire to determine functional capacity (the Duke Activity Status Index). Am J Cardiol. 1989;64:651-4

10. Alonso J, Permanyer-Miralda G, Cascant P, Brotons C, Prieto L, Soler-Soler J. Measuring functional status of chronic coronary patients. Reliability, validity and responsiveness to clinical change of the reduced version of the Duke Activity Status Index (DASI). Eur Heart J. 1997;18(3):414-9.

11. Mabo P, Victor F, Bazin P, et al. A randomized trial of long-term remote monitoring of pacemaker recipients (the COMPAS trial). Eur Heart J. 2011;33:1105-11. 
12. Brugada P. What evidence do we have to replace inhospital implantable cardioverter defibrillator followup? Clin Res Cardiol. 2006;95:III3-9

13. Heidbuchel H, Lioen P, Foulon S, Huybrechts W, Ector J, Willems R et al. Potential role of remote monitoring for scheduled and unscheduled evaluations of patients with an implantable defibrillator. Europace. 2008;10:351-7.

14. Santini M, Ricci RP, Lunati M, Landolina M, Perego $\mathrm{GB}$, Marzegalli $\mathrm{M}$ et al. Remote monitoring of patients with biventricular defibrillators through the CareLink system improves clinical management of arrhythmias and heart failure episodes. J Interv Card Electrophysiol. 2009;24:53-61

15. Masella C, Zanaboni P, Di Stasi F, Gilardi S, Ponzi $\mathrm{P}$, Valsecchi S. Assessment of a remote monitoring system for implantable cardioverter defibrillators. J Telemed Telecare. 2008;14:290-4.

16. Lunati M, Gasparini M, Santini M, Landolina M, Perego GB, Pappone C et al. Follow-up of CRT-ICD: implications for the use of remote follow-up systems. Data from the InSync ICD Italian Registry. Pacing Clin Electrophysiol. 2008;31: 38-46.

17. Nielsen JC, Kottkamp H, Zabel M, Aliot E, Kreutzer U, Bauer A et al. Automatic home monitoring of implantable cardioverter defibrillators. Europace. 2008; 10: 729-35.

18. Lazarus A. Remote, wireless, ambulatory monitoring of implantable pacemakers, cardioverter defibrillators, and cardiac resynchronization therapy systems: analysis of a worldwide database. Pacing Clin Electrophysiol. 2007;30:S2-12.

19. Neuzil P, Taborsky M, Holy F, Wallbrueck K. Early automatic remote detection of combined lead insulation defect and ICD damage. Europace. 2008;10:556-7.

20. Ricci RP, Morichelli L, Santini M. Remote control of implanted devices through Home Monitoring technology improves detection and clinical management of atrial fibrillation. Europace. 2009;11:54-61.

21. Spencker S, Coban N, Koch L, Schirdewan A, Muller D. Potential role of home monitoring to reduce inappropriate shocks in implantable cardioverter defibrillator patients due to lead failure. Europace. 2009;11:483-8.

22. Halimi F, Clémenty J, Attuel P, Dessenne X, Amara $\mathrm{W}$ on behalf of the OEDIPE trial Investigators. Optimized post-operative surveillance of permanent pacemakers by home monitoring: the OEDIPE trial. Europace. $2008 ; 10: 1392-99$.
23. Ricci RP, Morichelli L, D’Onofrio A, Calò L, Vaccari D, Zanotto G, et al. Effectiveness of remote monitoring of CIEDs in detection and treatment of clinical and device-related cardiovascular events in daily practice: the HomeGuide Registry. Europace. 2013;15(7):970-7.

24. Folino AF, Breda R, Calzavara P, Borghetti F, Comisso J, Iliceto S, et al. Remote follow-up of pacemakers in a selected population of debilitated elderly patients. Europace. 2013;15(3):382-7.

25. Schoenfeld SH, Compton SJ, Mead RH, Weiss DN, Sherfesee L, Englund J et al. Remote monitoring of implantable cardioverter defibrillators: a prospective analysis. Pacing Clin Electrophysiol. 2004;27:757-63.

26. Joseph GK, Wilkoff BL, Dresing T, Burkhardt J, Khaykin Y. Remote interrogation and monitoring of implantable cardioverter defibrillators. J Interv Card Electrophysiol. 2004;11:161-6.

27. Marzegalli M, Lunati M, Landolina M, Perego GB, Ricci RP, Guenzati G et al. Remote monitoring of CRTICD: the multicenter Italian CareLink evaluation- ease of use, acceptance, and organizational implications. Pacing Clin Electrophysiol. 2008;31:1259-64.

28. Fauchier L, Sadoul N, Kouakam C, Briand F, Chauvin $\mathrm{M}$, Babuty $\mathrm{D}$ et al. Potential cost savings by telemedicine-assisted long-term care of implantable cardioverter defibrillator recipients. Pacing Clin Electrophysiol. 2005;28:S255-9.

29. Raatikainen MJ, Uusimaa P, van Ginneken MM, Janssen JP, Linnaluoto M. Remote monitoring of implantable cardioverter defibrillator patients: a safe, timesaving, and cost-effective means for follow-up. Europace. $2008 ; 10: 1145-51$.

30. Zanaboni P, Landolina M, Marzegalli M, Lunati M, Perego GB, Guenzati G, et al. Cost-utility analysis of the EVOLVO study on remote monitoring for heart failure patients with implantable defibrillators: randomized controlled trial. J Med Internet Res. 2013;15(5):e106. 